\title{
Educación superior y TIC: conceptos y tendencias de cambio
}

\author{
Cristina LóPEZ-NAVAS \\ Universidad Complutense de Madrid \\ cristinalopeznavas@gmail.com
}

\begin{abstract}
Resumen:
El Espacio Europeo de Educación Superior propone la integración de las TIC en la educación superior como respuesta a las demandas de la sociedad del conocimiento. La literatura científico-técnica ha reflexionado sobre los fenómenos derivados de la presión tecnológica así como incorpora una terminología específica para nombrarlos. Con el propósito de ofrecer una visión general, este trabajo repasa esa literatura a través de una revisión de conceptos y proponiendo una clasificación de las principales tendencias de cambio en el sector educativo.
\end{abstract}

Palabras clave: Espacio Europeo de Educación Superior; TIC; presión tecnológica; conceptualización; literatura científico-técnica; Visión general

\section{Higher education and ITC: concepts and changing trends}

\begin{abstract}
:
The European Higher Education Space proposes the integration of ICT in higher education in response to knowledge society demands. Scientific and technical literature has reflected on the phenomena which derive from the technological pressure and incorporates a specific terminology to name them. The aim of this paper is to give an overall view of the mentioned literature, presenting a review of the concepts used and offering a classification of the main trends in education system changes.
\end{abstract}

Key Words: European Higher Education Space; ITC; technological pressure; conceptualization; scientific and technical literature; overall view

\section{Referencia normalizada:}

López Navas, C. (2014): Educación superior y TIC: conceptos y tendencias de cambio. Historia y Comunicación Social. Vol. 19. Núm. Especial Marzo. Págs. 227-239.

Sumario: 1. Introducción. 2. Metodología. 3. Los procesos de enseñanza-aprendizaje interdependientes de las TIC. 4. Las infraestructuras que están protagonizando los cambios educativos. 5. Los cambios en los sistemas educativos. 6. Conclusiones. 7. Propuestas.

\section{Introducción}

El Espacio Europeo de Educación Superior, ámbito de organización que se diseñó a finales de los noventa para armonizar los distintos sistemas educativos de la educa- 
ción superior europea, impone y exige acentuar y consolidar nuevos procesos de enseñanza-aprendizaje donde juega un importante papel la innovación tecnológica.

Más concretamente, las políticas educativas derivadas de este marco de actuación con frecuencia basan la orientación de sus actividades en la utilización de las infraestructuras comunicativas dentro y fuera del aula como garantía de progreso, bajo la premisa de que se trata de preparar a los estudiantes en el manejo de herramientas que les serán imprescindibles en su vida extramuros del centro educativo.

El optimismo que reside en esta apuesta se basa en el anhelo de la construcción de formaciones sociales que, TIC mediante, se configuren como "sociedades del conocimiento", a su vez superación de la "sociedad de la información" e íntimamente relacionadas con lo Manuel Castells acuñaría como "sociedad-red".

La literatura científico-técnica, por su parte, ha ido nutriendo estos propósitos de cambio de una nomenclatura específica para denominar los fenómenos derivados del uso de las TIC en educación, en general, y en la educación superior, en particular, pero también tratando de dar respuesta a los interrogantes que iban desafiando ese optimismo.

Entre otras cuestiones, esa literatura científico-técnica se pregunta por las consecuencias para el conjunto del sector (modelos, organización, protagonistas, etc.) que se derivan de una introducción -a veces descompensada respecto de otras condiciones y recursos- de esas infraestructuras tecnológicas en los sistemas educativos y sus dinámicas. Es decir, intentando analizar las correspondencias entre la intención de que las TIC mejoraran los procesos de enseñanza-aprendizaje y los resultados en la obtención del conocimiento o las competencias previstas.

Zawacki-Richter et al. (2009) -citados en Guri-Rosenblit y Gros (2011)- clasifican los estudios sobre estos fenómenos en tres niveles: micro, meso y macro. Repasando la literatura actual sobre el tema, se encuentran con alrededor de un $50 \%$ de la muestra de estudios micro centrados, principalmente, en los procesos de enseñanza-aprendizaje. Y muy pocos, y muy descriptivos, estudios meso y macro, referentes a la organización y gestión de la tecnología en las instituciones educativas, y a los marcos conceptuales de las teorías sobre los sistemas de educación basados en ella, respectivamente.

En este artículo, se analiza la creación, observación y reflexión por parte de científicos y técnicos como indagación preliminar para investigaciones posteriores que se ubiquen en la tercera línea de estudios que mencionaban los autores y autoras antes nombrados. A través de un repaso a la terminología utilizada para mencionar los cambios y las principales evoluciones que apuntan los textos en los sistemas educativos, se pretende obtener una visión general de la investigación actual sobre el tema, como condición primera para la construcción de marcos conceptuales que nos permitan abordarlo con perspectiva sistémica. 


\section{Metodología}

Para la consecución de los objetivos indicados en la introducción, se han efectuado dos tareas complementarias, de manera que el artículo queda dividido en dos partes.

En primer lugar, a través de investigación documental, se repasan los principales conceptos con los cuales se están describiendo los cambios en los sistemas educativos relacionados con la introducción de elementos comunicativos (principalmente tecnológicos). Con base en definiciones académicas que se han ofrecido sobre estas transformaciones, se ofrecen las claves comunicativas para la comprensión del fenómeno de la presión tecnológica a la que se está sometiendo a la educación, en general, y a la educación superior en particular.

En segundo lugar, a través de un análisis de contenido de una muestra de literatura científico-técnica sobre el tema, se presentan las tendencias de cambio en el sistema educativo relacionadas con los cambios infraestructurales que se están implementando en las comunicaciones. Esta segunda parte se basa en el trabajo que se viene desarrollando por el grupo de investigación "Identidades sociales y comunicación", del que formo parte, en el marco de la investigación "La producción social de comunicación y la reproducción social en la era de la globalización". Este equipo lleva a cabo una investigación empírica que, entre otros objetivos, se propone delimitar categorías de análisis para estudiar estas transformaciones con perspectiva socio-histórica.

En esta investigación concreta se han analizado textos que relacionan cambios en la comunicación y cambios en el resto de la sociedad. En estos textos se encuentran referencias a cómo se ve (o se verá) afectada la educación como consecuencia de la aparición y uso de tecnologías comunicativas. Los resultados del análisis se ofrecen clasificando los tipos de afectaciones que se pueden encontrar a todos los niveles del sistema educativo, teniendo en cuenta la modificación de las infraestructuras que se utilizan en los procesos educativos de la sociedad actual.

\section{Los procesos de enseñanza-aprendizaje mediados por TIC}

Si se definen los modos de interacción entre docente y discente como encuentros en los que los agentes intercambian información a propósito de unos temas con objeto de dar a conocer determinados contenidos o propiciar la reflexión sobre ellos, se pueden imaginar diferentes situaciones de enseñanza-aprendizaje y distintos repertorios de componentes necesarios para que se efectúen. En los modelos de educación presencial, estas interacciones se producen coincidiendo en espacios físicos y en tiempos. Y a ellos se pueden incorporar más o menos infraestructuras comunicativas en función de los objetivos del aprendizaje o según la presión tecnológica a la que se vean sometidos los agentes de la educación. 
Como alternativa a esta enseñanza-aprendizaje presencial, a lo largo del siglo $\mathrm{XX}$, ya se llevan a cabo muchas experiencias de educación a distancia. Las primeras formas tuvieron lugar en Europa e implicaban la utilización del correo postal para el intercambio del material formativo entre el profesor y el alumno. Esta comunicación se veía reforzada con el teléfono e incluso con tutorías presenciales. Estas experiencias educativas son los antecedentes de los actuales modelos de educación a distancia basados en TIC, los antecedentes de los fenómenos de e-learning.

\subsection{E-learning y B-learning}

La traducción literal del término e-learning a nuestra lengua es la de "aprendizaje electrónico". Dependiendo del punto de vista del autor, existen variadas definiciones del término, destacando una u otra faceta. Repasando la literatura se observa cómo el término e-learning se intercambia con el de d-learning (distance learning) para referirse a la educación a distancia en general, aunque se trate de un término derivado de la terminología moderna.

Mención aparte merece el b-learning, modalidad que pretende aunar lo mejor de lo presencial y lo mejor del e-learning. Son numerosas las maneras de abordarlo y también de denominarlo, pero en la mayoría de las ocasiones se refiere a: blended learning, aprendizaje semipresencial, o aprendizaje mixto/híbrido.

Otras expresiones que encierran el mismo significado que e-learning son, siguiendo a García Aretio (coord.), Ruiz Corbella, Domínguez Figaredo (2007): "educación virtual", "educación on-line", "e-formación" o "formación en espacios virtuales". Se utilizan indistintamente, aunque prevalezca el significante e-learning. En todo caso, cualquiera de estas variantes implica la "comunicación mediada por ordenador" o CMO.

Detrás de esos procesos se encuentran agentes educativos, profesores y estudiantes, que se ven sometidos a la presión tecnológica para desempeñar su trabajo de docentes los unos y corresponder a lo que se les exige como discentes los otros. Y lo mismo les pasa a las instituciones educativas, llevadas a aprender y a incluir las TIC en sus procesos de cambio.

\subsection{Los docentes y discentes que utilizan TIC para enseñar-aprender}

Desde hace algunos años se habla de "nativos digitales" como los estudiantes del mañana, que ya es hoy. Como indicaba Prensky (2001) a principios del milenio:

Los estudiantes universitarios de hoy representan las primeras generaciones formadas con las nuevas tecnologías. Han pasado sus vidas enteras rodeadas por el uso de computadoras, videojuegos, música digital, teléfonos móviles, y otros juguetes y herramientas de la era digital.

Por tanto, más de una década después, ya existen varias generaciones de nativos digitales que ocupan las aulas, físicas o virtuales que siguen teniendo maneras de 
aprender diferentes a las que tuvieron sus profesores (al menos de momento: inmigrantes digitales) y esa consecuencia se asocia a que crecieron manejando TIC. Estas diferencias generacionales van a hacer, además, replantear los roles de ambos en el proceso de enseñanza-aprendizaje, como veremos más adelante.

Por otro lado, para referirnos a los agentes colectivos, las instituciones de las que forman parte esos docentes y esos estudiantes, se encuentran también nuevas denominaciones. La clave para interpretar conceptos como "universidad virtual", "universidad 2.0", "universidad 3.0" está en la importancia que los actores le den a la presencia en el proceso de enseñanza-aprendizaje. Según esta importancia y la manera de asumirla, se desarrollan nuevos modos de procesar la educación superior que parecen querer acortar las diferencias clásicas entre la universidad a distancia y la universidad presencial.

Toda esta nueva terminología que se maneja al respecto de los cambios en la educación, es reflejo del enorme protagonismo que se le da a las infraestructuras comunicativas. En el siguiente punto se da cuenta de los conceptos genéricos y también se enuncian tecnologías concretas, de manera que se pueda ubicar en la historia de las tecnologías de la comunicación aquellos hitos que se marcan en el presente.

\section{Las infraestructuras que protagonizan los cambios educativos}

El sistema educativo se familiarizó con el acrónimo "TIC", que hace referencia a las Tecnologías de la Información y Comunicación. Se trata de un concepto dinámico que se construye a merced de cada autor o autora y cambia también según se producen cambios en la realidad que refleja. Ese dinamismo hace referencia a que, conforme los avances tecnológicos se producen, nuevas tecnologías van sustituyendo a otras anteriores, transformando la comunicación y los procesos sociales en los que se inserta.

Todas ellas son tecnologías que, en principio, facilitan los intercambios de información de los seres humanos. Son tecnologías porque son bienes diseñados y creados por la técnica, el "conjunto de procedimientos y recursos de que se sirve una ciencia o un arte", según la RAE. En este caso, de que se sirven las ciencias y el arte de la comunicación. Por lo tanto, Internet estaría incluido dentro de esta definición, aunque a veces se recurra a la expresión "TIC e Internet" para resaltar la importancia de la Red dentro de todas esas tecnologías que están revolucionando la existencia.

Hasta la aparición de la educación a distancia tradicional, la manera de comunicarse entre docente y discente era primordialmente verbal y a través de materiales textuales. Con la irrupción de las TIC, las vías de comunicación e intercambio de materiales formativos entre ambos se diversifican, modificando los espacios y los tiempos de la comunicación, así como la forma de los materiales que se intercambian. A continuación, proponemos una clasificación de TIC según la modificación en esos elementos del sistema comunicativo que introducen. 


\subsection{Infraestructuras que manipulan el tiempo comunicativo}

Para este primer punto, se toma la distinción de Granda (2008: 13), quien agrupa todas estas herramientas de comunicación entre profesores y estudiantes teniendo en cuenta las limitaciones temporales impuestas a los participantes del proceso de aprendizaje. Así, tendremos dos tipos de infraestructuras: a) aquellas que sirven de soporte a modelos asíncronos y b) aquellas otras que sirven a modelos síncronos:

- En los modelos asíncronos, entre el profesor y el alumno puede existir una separación tanto temporal como física. Son aplicaciones ejemplo de esta modalidad el correo electrónico, los blogs, o los foros. También se habla de soportes de modalidad asíncrona cuando se manejan CD-ROM o DVD.

- En la modalidad síncrona, entre el profesor y los alumnos existe una separación física, pero no temporal: docentes y discentes participan simultáneamente en el proceso de enseñanza-aprendizaje. Entre estos sistemas se encuentran los chats, los sistemas de videoconferencia, o tecnologías más específicas como las web de discusión de vídeo o los administradores de reuniones virtuales.

\subsection{Infraestructuras que modifican el espacio comunicativo}

En cuanto a los espacios, se observa cómo las aulas y los campus universitarios están siendo sustituidos en algunos casos y complementados en otros por espacios virtuales o en red. El apellido "virtual", acompañando en este caso a nombres que tradicionalmente denominan lugares físicos, refiere a una simulación, a una imitación de una realidad. La red Internet genera un ciberespacio, entendido como un conjunto de espacios virtuales, un ambiente electrónico donde ocurren procesos diversos y en los cuales participan actores que se valen de objetos y contenidos de información y conocimiento para realizar sus actividades. (Silvio, 1998).

Infraestructuras como ordenadores, tabletas, teléfonos inteligentes o cualquier dispositivo que pueda tener una conexión a Internet contribuyen a esa modificación de los espacios de la comunicación educativa. A este hardware se añade el software que se ha diseñado para mediar entre los agentes y las pantallas. Por ello merecen mención aparte las "aulas", "plataformas" y "campus" virtuales, como entornos en los que confluyen herramientas de todo tipo para mediar en la comunicación entre los agentes de la educación. El desarrollo de los mismos se ha producido sobre todo por su penetración en los niveles medios y superiores de la enseñanza, donde se ha apostado por ellos casi de forma incondicional.

\subsection{Infraestructuras que multiplican los lenguajes}

Por último, se recuerda que también los nuevos lenguajes son protagonistas de los cambios en la educación. A la palabra hablada se sumó la escrita, después la imagen fija, más tarde la imagen animada, los lenguajes audiovisuales... y las TIC, con sus códigos binarios, introducen nuevas maneras de dar forma a los mensajes: 
con imágenes en tres dimensiones (3D), con realidad virtual, con realidad aumentada, con realidad mixta, con lenguaje multimedia e hipermedia.

El hecho de que el lenguaje multimedia sea la combinación de varias formas expresivas (texto, imagen fija, imagen animada) da lugar a que algunos autores no lo consideren propiamente un lenguaje. Sin embargo, el hipertexto o el hipermedia sí son nuevas formas de presentación de los datos que se soportan en enlaces que van ampliando la información mostrada previamente. Introducen nuevas maneras de relación de la información cuando ésta es tratada como texto.

La realidad virtual, la realidad aumentada y la realidad mixta son sistemas tecnológicos basados en ordenadores y otros dispositivos de digitalización de imágenes cuyo fin es producir una apariencia de realidad que permita al usuario la sensación de estar presente en ella, ya sea con una inmersión total (realidad virtual) o parcial (realidad aumentada y mixta). Por último, se cuenta con los lenguajes $3 \mathrm{D}$, que directamente pretenden simular la visión humana real.

Con esta variedad de lenguajes se va dando forma, o, más técnicamente, "formato" a los materiales didácticos sobre los que se efectúa la acción docente. Se multiplican las herramientas que permiten crearlos: simuladores, programas de diseño de imágenes, de modificación de las mismas, aplicaciones, etc.

Con esta clasificación de las herramientas, según la modificación del tiempo y el espacio comunicativos o de la forma de los materiales que se intercambian, se refuerza la idea del protagonismo de los cambios comunicativos en las transformaciones de la educación. También hemos procurado apoyar esta idea repasando los conceptos que hacen referencia a cambios en los agentes, así como en los procesos de enseñanza-aprendizaje, en el ejercicio educativo mismo.

\section{Los cambios en los sistemas educativos}

Después de este esfuerzo de sistematización basado en análisis documental de aquellas modificaciones en los sistemas de comunicación que afectan o pueden afectar al sistema educativo, se procede a sistematizar los cambios en este último. Se ha optado por clasificar esas transformaciones según se indiquen afectaciones sobre los componentes del sector educativo, sus dinámicas y los modelos que rigen su funcionamiento.

El análisis se efectúa sobre una muestra de textos científico-técnicos en los que se mencionan TIC y cambios educativos. Algunas de las alusiones que se encuentran se refieren a la educación, en general. Se presentan aquí sin perder de vista que son referencias de esa índole, pero considerando que, desde un punto de vista macro-educativo, también se refieren a la educación superior, en particular. Se marcará en cada caso cuando las referencias encontradas estén centradas concretamente en ese nivel. 


\subsection{Transformaciones en el sector educativo}

Dos de los términos que se utilizan para referirse a los procesos de cambios en los que se ven envueltos el sistema y el sector educativos son: apertura y globalización. Ambas se basan en la posibilidad de acceso a la enseñanza desde cualquier lugar gracias a la innovación comunicativa. Esta posibilidad de acceso suele ser bien valorada en el contexto de las visiones optimistas sobre las innovaciones en el sector. Sin embargo, también aparecen voces críticas por parte de quienes encuadran tales transformaciones en el marco de la globalización económica y las alteraciones que ésta produce sobre la educación.

Desde esas perspectivas críticas con la globalización económica, se encuentran referencias a la desregulación del sector educativo vinculada al uso de las TIC. Hay autores que advierten de la sustitución del sistema educativo por el sistema de comunicación, con el argumento de que éste último asimila e integra las funciones y los recursos educativos y propicia el traslado de la educación al ámbito de las actividades productivas para el capital.

Los textos que abundan más en esta perspectiva advierten de una pérdida de sentido de la inversión en educación por el uso pedagógico de los medios de comunicación, cuando este uso llega a cuestionar el proceso educativo de la enseñanza-aprendizaje en los espacios físicos. A estos juicios se añaden otros que señalan el sometimiento al mercado de los educadores, quienes se verían convertidos en agentes de ventas.

Las referencias a la educación superior, en particular, concretan los procesos previamente indicados en un aumento de profesionales del conocimiento y en la aparición y consolidación de nuevos equipos de trabajo multidisciplinares compuestos por expertos en distintos saberes, además de por pedagogos y técnicos. Todas estas presiones productivas y tecnológicas han llevado, además, a la aparición de nuevas organizaciones de gestión y acreditación de la enseñanza superior.

Por último, cabe destacar otro grupo de referencias a los cambios en las circunstancias en las que se desarrolla la educación con alusiones a los nuevos escenarios para la enseñanza. En esta línea, hay autores que apuntan a la pérdida de distinción entre espacios domésticos, laborales, lúdicos y formativos y, yendo más allá, a la integración en un único microsistema del espacio doméstico, el laboral y el formativo.

\subsection{Modificaciones en las dinámicas educativas}

En cuanto a las prácticas o actividades de la educación los textos analizados dejan constancia de la complicación y el cuestionamiento al que se ven sometidas con cada innovación comunicativa. Tanto en los procesos de enseñanza-aprendizaje como en otras dinámicas de la educación superior (la investigación y la capacitación docente entre ellas) se encuentran referencias contradictorias.

Quienes tienen una visión más positiva recogen cada modificación como novedad y como fortaleza. En el caso concreto de la educación superior, el optimismo se basa, 
por ejemplo, en la integración internacional, que se desarrolla sustentada en prácticas de tele-educación.

Dentro de estas visiones, los textos también señalan rupturas con la linealidad y la pasividad del aprendizaje en la educación superior, así como con la jerarquía, verticalidad y autoritarismo en la transmisión del saber.

Apuntan de la misma forma a un aumento de las prácticas colaborativas a todos los niveles y la aparición de nuevas dinámicas educativas basadas en el constructivismo social, incluso al desarrollo de la educación-red.

Como visiones más críticas, se encuentran aquellas que señalan una reducción de posibilidades de aprendizaje de los ciudadanos provocada por la dificultad de acceso a sus soportes y por la continua innovación en los mismos, dinámicas relacionadas con el fenómeno de la brecha digital, extensivas a la educación superior.

También se hallan referencias a tendencias de individualización en el estudio, mecanización de las formas de aprender, y a una deshumanización de los procesos de enseñanza provocada por la mediatización tecnológica de los procesos.

Por último, se advierte del distanciamiento entre los procesos de aprender y aprehender como consecuencia del uso sostenido en el tiempo de los buscadores como única herramienta de acceso a la información, lo cual modifica la percepción del aprendizaje como una actividad que se daría por completa por las búsquedas ejecutadas por los estudiantes.

Todas estas tendencias en las prácticas educativas imponen un refuerzo de la alfabetización funcional y la necesidad de alfabetización digital u otras formas de alfabetización, de lo cual también se encuentran referencias en los textos. El fracaso escolar causado por el ocio mediático es otro de los riesgos socio-educativos que se advierten en la literatura científico-técnica, fracaso que estaría relacionado a su vez con la disminución de pensamiento crítico.

En cuanto a las otras dinámicas de la educación superior, los textos declaran una reactivación de los mecanismos de trabajo multidisciplinario en investigación. Estos procesos se verían dinamizados por las nuevas infraestructuras comunicativas, para cuya utilización los autores señalan el desarrollo de programas de capacitación docente.

\subsection{Cambios en los modelos educativos}

En este apartado recogemos alusiones a valores y modelos educativos así como a objetivos, perspectivas para crear materiales didácticos y diseños formativos, y también las referencias a los roles de los agentes del proceso de enseñanza-aprendizaje, y a los criterios de calidad que se establecen para el control del funcionamiento del sistema.

En cuanto a los valores, se encuentran enunciados sobre la permanencia de todos los niveles axiológicos sobre los que se fundamenta la educación superior, así como 
se observa continuidad de valores intrínsecos de la educación como el respeto y la responsabilidad.

En segundo lugar, se observa cómo la literatura científico-técnica también reflexiona sobre los modelos pedagógicos para la enseñanza superior, insistiendo, con diferentes grados de intensidad, en el tipo de cambio que acontece y en lo que se mantiene.

Se recoge una continuidad en los modelos de aprendizaje de saberes aplicados, así como en los modelos basados en la construcción colectiva del conocimiento y en el modelo de aprendizaje a lo largo de la vida (long life learning).

Por otro lado, se hace alusión, de manera general, a la aparición de un nuevo paradigma educativo con nuevos modelos pedagógicos a todos los niveles, también en la educación superior. Más concretamente, se alude a un cambio de propuestas educativas que rompan con la linealidad de los procesos de enseñanza-aprendizaje clásicos y a una ruptura con el modelo jerárquico de transmisión de conocimientos.

En otros casos, se anuncia una evolución de los modelos de educación a distancia y una desaparición de los modelos tradicionales de este tipo, sobre todo en la educación de tercer ciclo.

Todas estas modificaciones en los modelos formales son lo que unos autores y otros indican como lo que debería ser el proceso de enseñanza-aprendizaje. En algunos casos van, además, acompañadas de objetivos concretos, como el "aprender a aprender" y aprender a enfrentarse a la búsqueda de información, siempre ligados al desarrollo de la sociedad del conocimiento.

En esta línea de ideas sobre las que construir la educación se encuentran referencias a modos de diseñar la formación y los materiales didácticos. Se podría decir que se trata de propuestas para que esos modelos que se nombran puedan regir en la práctica. Así, la literatura científico técnica que une los cambios en la comunicación con los cambios en la educación anuncia una necesaria evolución de los diseños formativos.

La sustitución progresiva de diseños de instrucción basados en teorías conductistas por teorías constructivistas es un ejemplo de lo que los textos proponen. En cuanto a la proyección de materiales didácticos, se menciona la adopción de una perspectiva multidisciplinar, así como el aprendizaje significativo como base de los nuevos recursos educativos de la enseñanza superior.

Como control de estas propuestas teóricas, los textos también recogen la necesidad de establecer criterios, estándares e indicadores de calidad de la enseñanza superior, si bien no se alude a ninguno en concreto en la muestra.

Por último, dentro de este bloque de valores, modelos, ideas que mueven o paralizan los cambios en la educación se señala un cambio en los roles de los agentes del proceso educativo. De manera general, se señala la conversión del profesor en un agente coordinador y creador de situaciones de enseñanza. 
Explícitamente se señala que los profesores de la enseñanza superior deben asumir los objetivos mencionados más arriba como los de su acción didáctica para asistir en la búsqueda de información. Y, más concretamente, se invita a un cambio en los roles del tutor, cuyo protagonismo deducimos que aumenta con la necesidad de seguimiento que precisan los modelos antes enunciados.

\section{Conclusiones}

Como se extrae de la segunda parte del artículo, la percepción del impacto que las TIC están teniendo en la educación (superior) en la literatura científico-técnica no es necesariamente negativa ni positiva.

Existen voces que anuncian los problemas derivados de la presión tecnológica a la que se está sometiendo al sistema educativo, así como existen reflexiones optimistas sobre las transformaciones que acontecen. Alertan, sobre todo, las tendencias a la mercantilización del sector educativo y las identificaciones de la educación con mera comunicación. Sin embargo, esperanzan las posibilidades de apertura y de traspaso de todo tipo de fronteras.

Respecto de las dinámicas, también los textos encuentran luces y sombras. Las primeras se fundan en las potencialidades del trabajo en grupo, tanto a nivel del proceso de enseñanza-aprendizaje como en investigación. Por el contrario, las advertencias de las consecuencias negativas señalan los riesgos de aislamiento provocados por el uso individual de la mayoría de las tecnologías, así como la brecha digital, únicamente evitable con la extensión de la alfabetización, funcional primero y digital después.

El tercer bloque de menciones, las referentes a las transformaciones en la manera de pensar la educación, se contempla como las respuestas que las ciencias de la educación ofrecen para hacer frente a los nuevos retos que las tecnologías proponen. Y también se identifican contraposiciones a este nivel, encontrando literatura que apuesta por el mantenimiento de los valores educativos y de los modelos pedagógicos sobre los que ya pivotaba la interacción docente-discente, así como textos cuyas posturas teóricas son más revolucionarias.

\section{Propuestas}

La reflexión teórica sobre educación y TIC parece girar en torno a la idea del protagonismo que se otorga a las infraestructuras comunicativas en las actuales acciones pedagógicas. Queda también reflejada en los textos la creación y extensión de una nomenclatura que nombra los nuevos fenómenos que surgen de esas sinergias. 
No corresponde a un análisis de este tipo llegar a conclusiones prácticas para que los procesos de enseñanza-aprendizaje se vean enriquecidos, o no, con tecnologías de la comunicación, pero sí cabe proponer nuevas líneas de análisis que completen las que se encuentran ahora disponibles para la reflexión teórica sobre estos temas.

Se recuerda que los objetivos de estas páginas apuntaban a la obtención de una visión general de estos fenómenos, planteando la posibilidad de encontrar fallas de conocimiento sobre las que investigar que ayuden al desentramado de su complejidad. Se considera, por tanto, que serían oportunos todos aquellos estudios que analizaran la literatura científico-técnica producida a propósito de un nivel particular. Por ejemplo, un meta-análisis de la investigación sobre infraestructuras que modifican el espacio comunicativo y su relación con los cambios en los roles de los agentes del proceso de enseñanza-aprendizaje.

La presión tecnológica a la que se somete a la educación aumenta a medida que se refuerzan las políticas educativas y se extiende la asimilación de los nuevos conceptos. Por eso se recalca la idea de que es preciso contemplar los cambios desde una distancia teórica y trabajar en la construcción de marcos conceptuales e indicadores para estudiarlos.

\section{Bibliografía}

GARCÍA ARETIO, Lorenzo. (Coord.); RUIZ CORBELLA, Marta; DOMÍNGUEZ FIGAREDO, Daniel. (2007). De la educación a distancia a la educación virtual. Ariel. Barcelona.

GRANDA CANDÁS, Juan Carlos (2008): Caracterización, evaluación y optimización de sistemas multimedia interactivos en entornos de e-learning síncrono. Tesis para la obtención del grado de Doctor. Universidad de Oviedo.

GURI-ROSENBLIT, Sarah; GROS, Begoña. (2011): "E-Learning: Confusing Terminology, Research Gaps and Inherent Challenges", en The Journal of Distance Education / Revue de l'Éducation à Distance, volumen 25, $\mathrm{n}^{\circ} 1$. Disponible en: http://www.jofde.ca/index.php/jde/article/view/729/1206 [29-08-2013]

PRENSKY, Marc (2001): Nativos digitales, inmigrantes digitales. On the Horizon, MCB University Press, Vol.9, $\mathrm{n}^{\circ} 5$, oct. 2001. Traducción libre, en revisión, a cargo de Mara Vanina Osés, con la colaboración de Pamela Morelli. Disponible en: http://psiytecnologia.files.wordpress.com/2010/02/prensky-nativos-digitales-inmigrantes-digital-traduccion.pdf [24-09-2013]

SILVIO, José (1998): "La virtualización de la educación superior: alcances, posibilidades y limitaciones", en Educación Superior y Sociedad, vol. 9, nº 1: 27-50. Editada por UNESCO-IESALC, 1998. 


\section{La autora}

Cristina López-Navas es doctoranda en el programa "Comunicación, Cambio Social y Desarrollo" de la Universidad Complutense de Madrid. Investigadora del programa de Formación de Profesorado Universitario FPU. Miembro del Grupo de Investigación UCM "Identidades Sociales y Comunicación”. Licenciada en Periodismo por la Universidad San Pablo CEU de Madrid y Máster en Comunicación Social por la Universidad Complutense de Madrid. 